\title{
Pneumothorax after insertion of central venous catheters in the intensive care unit: association with month of year and week of month
}

\author{
Najib T Ayas, Monica Norena, Hubert Wong, Dean Chittock, Peter M Dodek
}

Qual Saf Health Care 2007;16:252-255. doi: 10.1136/qshc.2006.021162

See end of article for authors' affiliations

Correspondence to: Dr P Dodek, Center for Health Evaluation and Outcome Sciences, 1081 Burrard Street, Vancouver, British Columbia, Canada V6Z 1Y6; pedodek@ interchange.ubc.ca

Accepted 11 March 2007
Rationale: One of the complications associated with insertion of central venous catheters (CVCs) is pneumothorax (PTX). Because of housestaff inexperience, it was hypothesised that rates of PTX after insertion of CVCs in teaching hospitals would be highest in July and August and in the first week of the month (beginning of intensive care unit (ICU) rotation).

Methods: In a retrospective analysis of data from patients admitted to the ICU in two tertiary care teaching hospitals in British Columbia from 1999 to 2005, rates of PTX occurring after insertion of CVCs were calculated, and it was evaluated whether rates were increased during certain times of the year/month. Results: During this period, 3548 patients were admitted to these ICUs and had at least one CVC placed. 5816 CVCs were inserted; 113 PTX occurred within 2 days after insertions (1.9\% per CVC). The rate during the last week of the month was greater $(2.7 \%)$ than during the first, second or third weeks $(1.7 \%, 1.8 \%$ and $1.4 \%$, respectively). This effect persisted after controlling for the Acute Physiology and Chronic Health Evaluation II score, the number of catheters placed per patient, gender, age and hospital. Rates of PTX after catheter placement did not vary by the month of the year.

Conclusions: The rate of PTX affer insertion of CVCs is greatest in the last week of the month. If this effect can be verified in other centres, increased supervision of residents at the end of ICU rotations when placing CVCs should be considered. Whether this effect applies to other patient safety outcomes in the ICU also needs further study.
$\mathrm{T}$ e insertion of central venous catheters (CVCs) is a common invasive procedure performed in the intensive care unit (ICU). ${ }^{1}$ CVCs are inserted percutaneously through large veins such as the subclavian, jugular and femoral veins. Although they may assist in the monitoring and treatment of patients who are critically ill, CVCs are associated with potentially serious complications including infection and thrombosis. ${ }^{2}$ One of the major complications associated with insertion of CVCs is pneumothorax (PTX) due to inadvertent puncture of the lung at the time of inserting a needle into a large vein.

In academic teaching hospitals, most CVCs are inserted by housestaff (residents, interns and fellows). The effects of housestaff scheduling on rates of occurrence of PTX after insertion of CVCs has not been well studied. The purpose of this study was to determine whether the rate of occurrence of PTX after insertion of CVCs is related to the month of the year or the week of the month. We hypothesised that the rate of occurrence of PTX after CVC placement in teaching hospitals would be greatest at the beginning of the academic year (July and August) and in the first week of the month (beginning of the ICU rotation) because of inexperience of housestaff during these time periods.

\section{MATERIALS AND METHODS Database}

The ICU database used in this study has been the repository for clinical ICU data from two tertiary care teaching hospitals (St Paul's Hospital and Vancouver General Hospital, Vancouver, British Columbia, Canada) since October 1998. Details of the database have been described previously. ${ }^{3}$ We used data from patients admitted to these two ICUs between January 1999 and December 2005. The database was comprehensive and included the following information: hospital admission/discharge dates, ICU admission/discharge dates, primary ICU-admitting diagnosis, other ICU-admitting diagnoses, underlying diagnoses, ICU-acquired diagnoses, Acute Physiology and Chronic Health Evaluation (APACHE) II score, procedures performed in ICU, complications in ICU, hospital mortality, hospital length of stay and ICU length of stay. This information was entered by specially trained ICU nurses who reviewed all ICU charts concurrently and were unaware of the hypotheses for this study.

Diagnosis fields were populated using the ICNARC (Intensive Care National Audit \& Research Centre) coding method (http:// www.icnarc.org/audit/cmp/icm).

Accuracy of data was increased by automatic alerts for nonsense values and electronic calculation of severity scores after manual input of the elements of these scores. Having 10 different individuals entering data over the 7 years that we have had this database has required intermittent qualitative tests of inter-rater and intra-rater reliability using samples of records; these tests of reliability have always been satisfactory.

\section{Description of hospitals}

The ICU at St Paul's Hospital, Vancouver, Canada, is a 13-bed medical-surgical unit within a 400-bed tertiary care, teaching hospital.

The ICU at Vancouver General Hospital is a 27-bed medicalsurgical-trauma unit within a 600-bed tertiary care teaching hospital. There are separate cardiac surgical and cardiac care

Abbreviations: APACHE, Acute Physiology and Chronic Health Evaluation; CVC, central venous catheter; ICU, intensive care unit; PTX, pneumothorax 


\section{Table 1 Patients' demographics}

\begin{tabular}{ll}
\hline N & 3548 \\
Mean (SD) age (years) & $57.3(17.3)$ \\
Gender (\% male) & 54.7 \\
Mean (SD) APACHE II score & $23(7.0)$ \\
& \\
Hospital mortality (\%) & \\
Top 10 primary ICU admitting diagnoses, n (\%) & $427(12.0)$ \\
$\quad$ Septic shock & $300(8.4)$ \\
$\quad$ Bacterial pneumonia & $253(7.1)$ \\
$\quad$ Septicemia & $168(4.7)$ \\
Inhalation pneumonitis (gastrointestinal contents) \\
Non-cardiogenic pulmonary oedema (ARDS) & $145(4.1)$ \\
Cardiogenic shock & $140(3.9)$ \\
Primary brain injury & $131(3.7)$ \\
$\quad$ Cardiogenic pulmonary oedema & $78(2.2)$ \\
Exacerbation of chronic obstructive airway disease & $75(2.1)$ \\
Pneumonia, no organism identified & $61(1.7)$ \\
& $1778(43.2)$ \\
Source of ICU admission & \\
Another ward & \\
Another hospital & 2702 \\
Emergency & 438 \\
& 405 \\
Total & \\
\hline
\end{tabular}

APACHE, Acute Physiology and Chronic Health Evaluation; ARDS, acute respiratory distress syndrome; ICU, intensive care unit.

units at each of these institutions. These ICUs are staffed by board-certified intensivists who rotate every week.

Procedures are performed predominantly by junior and senior residents rotating on the ICU service. Junior residents are supervised by senior residents. There is no formal period of time in which junior residents are prohibited from performing procedures. Each unit is staffed by at least one ICU fellow and several senior and junior residents. ICU attendings and fellows are present in the ICU and supervise procedures during daylight hours every day of the week. Fellows are often present in the ICU during the early evenings. However, attendings and fellows only rarely stay in the hospital overnight.

Residents usually begin rotations on the first of every month, with durations of service ranging from 1 to 2 months. From 2002 to $2005,70 \%$ of rotations were 1 -month rotations, and $78 \%$ of residents began their rotations during the first week of each month.

\section{Ascertaining the number of CVCs inserted}

The database contains information on all invasive procedures performed in the ICU, including insertion of CVCs. The following procedures that were coded in the database were considered CVCs and included in our analyses: internal jugular, subclavian, dialysis catheter insertion, pulmonary arterial catheter, jugular venous bulb catheter, CVC and transvenous pacemakers. When multiple catheters were reportedly inserted into a patient on the same day, they were carefully reviewed to avoid reporting the same CVC twice. Only new insertions (and not rethreading of CVCs) were included.

CVCs placed outside of the ICU (eg, emergency department, surgical suite) were not recorded in the database. The anatomical location (ie, subclavian, jugular and femoral) and date of the CVC insertion were available. For this analysis, we only included CVCs inserted in the subclavian or internal jugular vein as femoral CVC insertions are not associated with PTX.

\section{Ascertaining PTX associated with CVC placements}

For our analysis, we defined CVC-related PTX as any PTX recorded as an "ICU-acquired diagnosis" or as a "complication" occurring within $48 \mathrm{~h}$ after a CVC was inserted in either the subclavian or the jugular vein.

We excluded patients in whom PTX was a "primary ICUadmitting" or "other ICU-admitting diagnosis", and who had an ICU-acquired diagnosis of PTX and documentation of a CVC insertion on the day of admission to the ICU. For these patients, it would have been difficult to distinguish a PTX that was part of the admitting problem (and unrelated to a CVC insertion) from a PTX that occurred after insertion of a CVC.

\section{Statistical analysis}

For each month, the number of CVCs inserted was tabulated. The crude rate of CVC-associated PTX was defined as the number of PTX that occurred after the insertion of CVCs divided by the total number of CVCs inserted that month.

Each month was then divided into 4 weeks - that is, days 1-7 of the month was reported as week 1, days 8-14 as week 2, days 15-21 as week 3 and day 22 until the end of each month as week 4. Numbers of CVCs inserted in the first, second, third and fourth weeks of all months were tabulated, and rates of PTX were calculated for each of these weeks.

For our analysis, it was necessary to identify the date of the CVC responsible for the PTX. Therefore, if a patient had more than one CVC placed, we assumed that the CVC placed closest to the date of the PTX was responsible for the incident.

Logistic generalised estimating equation models (with multiple CVC insertions in a patient treated as a cluster) were used to determine whether month of the year or week of the month was independently associated with the occurrence of a PTX after adjusting for age, gender, APACHE II score and ICU site.

This observational study was approved by the Research Ethics Board of Providence Health Care-there was no requirement for informed written consent.

\section{RESULTS}

From January 1999 to December 2005, 8077 patients were admitted to these two ICUs. A total of 388 patients were excluded from the analysis; 383 because of missing variables (predominately APACHE score) and 5 because PTX was a primary ICU-admitting or other ICU-admitting diagnosis, and had an ICU-acquired diagnosis of PTX and a CVC insertion on the day of admission. Of the 7689 remaining patients, 3548 patients had at least one CVC placed during their ICU stay and were considered as the study cohort.

Table 1 shows the demographics of the patients included in the study. The mean age of these patients was 57.3 years. A total of 5816 CVCs were placed. These included 2524 internal jugular, 1522 subclavian, 791 dialysis catheters, 593 coded as

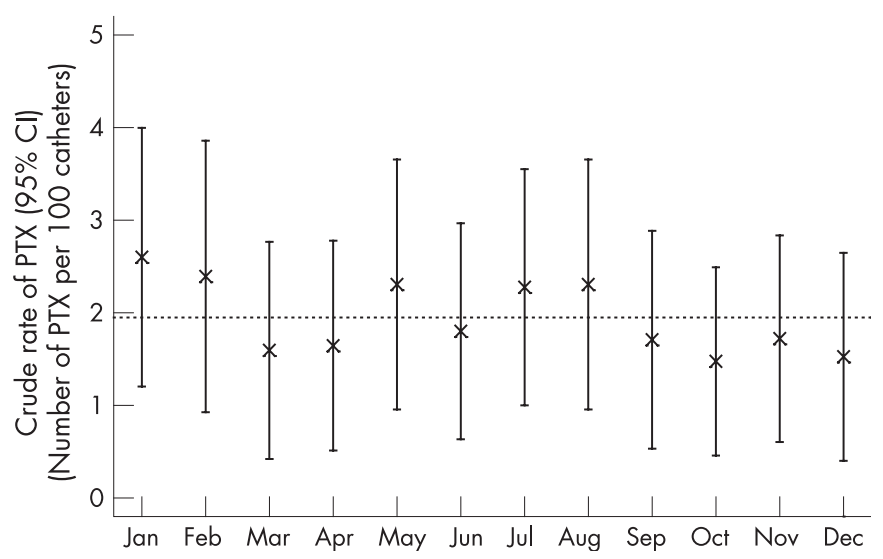

Figure 1 Crude rate of pneumothorax (PTX; \%) and $95 \% \mathrm{Cl}$ according to the month of the year. 


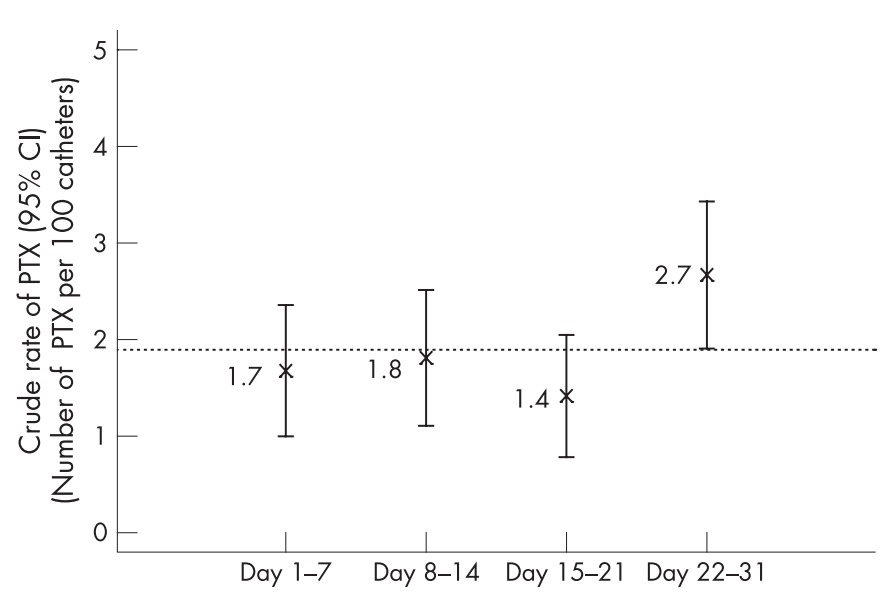

Figure 2 Crude rate of pneumothorax (PTX; \%) and 95\% Cl according to the week of the month.

CVCs, 326 pulmonary arterial catheters and 60 transvenous pacemakers. In all, 113 PTX occurred after these procedures ( crude rate $=1.9 \%$ PTX/CVC placed).

Figure 1 shows the rate of PTX (per CVC) for each month. There was no significant relationship between the rate of PTX and the month of the year. In particular, rates of PTX were not increased at the beginning of the academic year compared with the end of the year. That is, rates in July and August were $2.3 \%$ and $2.3 \%$, respectively, compared with $2.3 \%$ and $1.8 \%$ in May and June ( $\mathrm{p}=0.77$, July and August vs other months, $\chi^{2}$ test).

We then carried out a multivariate regression analysis (generalised estimating equations) to assess whether rates in July and August were greater than the rates in the other months after controlling for age, gender, APACHE II score and hospital site. There was still no significant effect of month (OR $1.24,95 \%$ CI 0.79 to $1.97, \mathrm{p}=0.35$ ).

However, the rate of PTX varied by the week of the month (fig 2). Specifically, during the last week of the month, the rate was greater $(2.7 \%)$ than that during the first, second or third weeks $(1.7 \%, 1.8 \%$ and $1.4 \%$, respectively). This effect persisted after controlling for APACHE II score, gender, age and hospital site (OR 1.6 and 95\% CI 1.1 to 2.4 for the fourth week compared with the other three weeks).

A total of 2246 patients had only one CVC placed. The rate of PTX was $1.7 \%$ for those who had only a jugular catheter and $1.5 \%$ for those who had only a subclavian catheter.

\section{DISCUSSION}

In our study, rates of PTX after insertion of CVCs did not vary by the month of the year, but did vary by the week of the month. Specifically, PTX after CVC insertion was more common in the last week of the month than in the first week of the month. This effect persisted after controlling for a variety of potential confounders.

These findings were surprising. We hypothesised that rates of PTX would be greatest at the beginning of the month (ie, start of the ICU rotation for most housestaff), and believed that rates would decrease as the rotation progressed, as housestaff would presumably became more comfortable and competent at performing procedures.

The explanation for the increased rate of PTX at the end of the rotation is open to speculation. One possibility is that there may be greater supervision of housestaff at the beginning of the rotation by fellows and staff than at the end of the rotation. Also, residents may become more aggressive or overconfident in the insertion of CVCs as they gain general experience, leading to multiple passes with the needle and a consequently greater rate of PTX. Third, ICU rotations tend to have frequent overnight shifts, leading to chronic sleep deprivation. ${ }^{4}$ Given that fatigue adversely affects motor coordination, ${ }^{6}$ increased fatigue by the end of the rotation may be associated with increased rates of procedural complications. Fourth, it is possible that senior residents may have performed more procedures at the beginning of the month, rather than at the end of the month, whereas the opposite was true for junior residents.

Because we did not collect information regarding these variables (eg, subjective or objective measures of fatigue, number of CVCs placed by each resident, extent of supervision during the procedure, level of experience of operator/supervisor), we do not know which (if any) of these might account for our findings. Future prospective studies that collect this information may provide us with greater insight into how to reduce these complications in the future.

A recent comprehensive study of serious medical errors in the ICU has shown that $75 \%$ of incidents occur during treatments or procedures. ${ }^{7}$ Our findings suggest that resident scheduling affects rates of these complications. Although we have potentially demonstrated this for only one safety outcome, rates of other procedure-related complications may be similarly affected. If this is the case, interventions designed to provide closer supervision of residents at the end of their rotation may substantially improve patient safety.

The lack of a relationship between the month of the year and rates of PTX was also surprising. One potential possibility for the lack of relationship may be increased supervision of residents by staff and fellows at the beginning of the academic year, thus counterbalancing the inexperience of residents at this time. This would be consistent with other studies that have demonstrated no reduction in the quality of care in patients admitted to teaching hospitals in July, ${ }^{8}$ and no increase in mortality in patients admitted to the ICU in July.

Our study has several strengths. First, it was based on $>3500$ patients admitted to mixed medical-surgical ICUs in two hospitals over 7 years. The lack of selection bias and the similarity of our patient population to that of other ICUs probably improves the generalisability of our findings to other similar ICUs. Second, we used multivariate analyses to determine the independent effects of the month of the year, and the week of the month. Specifically, we adjusted for potential confounders, including age, gender, severity of illness, hospital site and numbers of CVCs placed.

We acknowledge that there are a number of limitations to our study. First, it is a study based on only two tertiary ICUs in a single geographical area; these findings may not apply to nontertiary care ICUs or to specialty ICUs that cater to a specific group of patients. Second, approximately $20 \%$ of residents did not start their ICU rotations during the first week of the month. As such, the effect we observed may be an underestimate of the true effect of the week of rotation. Also, data regarding duration and start date were based on data from 2002 to 2005, rather than from 1999 to 2005. However, there have been no substantial changes in these logistics for many years, and we are thus confident that these descriptors are accurate for the period of this study as well. Third, we may not have captured all PTX. We doubt that this would be the case as our rate of PTX was slightly greater than that reported by others. ${ }^{10}$ In addition, even if some PTX were missed, it is unlikely that the probability that a PTX was missed would vary by the month or by the week of the month. Fourth, ultrasound guidance was not used consistently in our ICUs for insertion of catheters during this time period. This technology reduces the risk of PTX, ${ }^{11}$ and regular use of this technology might have modified our results. In these two ICUs, ultrasound was used more often during the last few years of the study, and was not used consistently 
before this time. Indeed, consistent with this concept, rates of PTX were greater during the first 4 years (1999-2002) of the study $(2.55 \%)$ than during the last 3 years $(1.46 \%)$. Fifth, on the basis of the language used to describe catheters in our database, we cannot be certain that all of the catheter insertions were in one of the internal jugular or subclavian veins. Specifically, it is possible that some of the pulmonary arterial catheters, or pacemakers, or "CVCs" were inserted in the femoral vein. However, on the basis of our own observations of practice in these ICUs, we believe that most of these catheters would have been placed in one of the neck veins. Sixth, the fourth week included events that occurred from the 22 nd day to the end of the month. Although we acknowledge that this would include a larger number of days than the other weeks, this should not affect the rate of PTX per CVC placement. Finally, because our analysis was based on a retrospective post hoc analysis of an audit database, we lacked information about some potentially important confounders. For instance, we did not have information about the person attempting insertion (ie, the experience and level of training of the resident), the degree of supervision, time of procedure, the difficulty, number of passes with the needle and whether ultrasound was used for a particular CVC placement.

Because of these limitations, we acknowledge that our results should be considered more "hypothesis-generating" as opposed to definitive. Nevertheless, we believe that our study is useful because it highlights the need to examine and audit the performance of residents when they are performing procedures with potentially significant complications.

\section{CONCLUSION}

This retrospective study suggests that the rate of PTX after insertion of CVCs is highest during the last week of the month. Increased supervision of residents at the end of ICU rotations when placing CVCs should be considered. Whether this effect applies to other patient safety outcomes in the ICU needs further study.

\section{Authors' affiliations}

Najib T Ayas, Peter M Dodek, Program in Critical Care Medicine, Providence Health Care, Vancouver, British Columbia, Canada Monica Norena, Hubert Wong, Center for Health Evaluation and Outcome Sciences, Vancouver, British Columbia, Canada
Dean Chittock, Critical Care Medicine, Vancouver General Hospital, Vancouver, British Columbia, Canada

Najib T Ayas, Dean Chittock, Centre for Clinical Epidemiology and Evaluation, Vancouver Coastal Health Research Institute, Vancouver, British Columbia, Canada

Najib T Ayas, Respiratory Division, Vancouver General Hospital,

Vancouver, British Columbia, Canada

Najib T Ayas, Dean Chittock, Peter M Dodek, Department of Medicine, University of British Columbia, Vancouver, British Columbia, Canada

Funding: This work was supported by the BC Health Research Foundation, the Keenan Foundation and the Michael Smith Foundation for Health Research (Infrastructure Grant-ICU Patient Safety Team). NTA was supported by a Michael Smith Foundation Health Research Scholar Award, a Departmental Scholar Award from the Department of Medicine $\mathrm{UBC}$ and a CIHR/BC Lung Association New Investigator Award.

Competing interests: None.

\section{REFERENCES}

1 Mermel LA, Farr BM, Sherertz RJ, et al. Infectious Diseases Society of America; American College of Critical Care Medicine; Society for Healthcare Epidemiology of America. Guidelines for the management of intravascular catheter-related infections. Clin Infect Dis 2001;32:1249-72.

2 Ruesch S, Walder B, Tramer MR. Complications of central venous catheters: internal jugular versus subclavian access - a systematic review. Crit Care Med 2002;30:454-60.

3 Norena M, Wong $\mathrm{H}$, Thompson WD, et al. Severity of illness and comorbidity scores as predictors of intensive care outcomes. J Crit Care 2006;21:142-50.

4 Lockley SW, Cronin JW, Evans EE, et al. Effect of eliminating extended duration work shifts and reducing weekly work hours on sleep and attentional failures in interns. N Engl J Med 2004;351:1829-37.

5 Ayas NT, Cronin JW, Barger LK, et al. Self-reported medical errors, needlesticks, and stress in the ICU versus the hospital ward: results of a national survey of US interns [abstract C24]. Proc Am Thor Soc 2005:A595.

6 Grantcharov TP, Bardram L, Funch-Jensen P, et al. Laparoscopic performance after one night on call in a surgical department: prospective study. BMJ 2001;323:1222-3.

7 Rothschild JM, Landrigan CP, Cronin JW, et al. The Critical Care Safety Study: the incidence and nature of adverse events and serious medical errors in intensive care. Crit Care Med 2005;33:1694-700.

8 Rich EC, Gifford G, Luxenberg M, et al. The relationship of house staff experience to the cost and quality of inpatient care. JAMA 1990;263:953-7.

9 Barry WA, Rosenthal GE. Is there a July phenomenon? The effect of July admission on intensive care mortality and length of stay in teaching hospitals. J Gen Intern Med 2003;18:639-45.

10 Ruesch S, Walder B, Tramer MR. Complications of central venous catheters: internal jugular versus subclavian access-a systematic review. Crit Care Med 2002;30:454-60.

11 Hind D, Calvert N, McWilliams R, et al. Ultrasonic locating devices for central venous cannulation: meta-analysis. BMJ 2003;327:361. 